DOI: https://doi.org/10.11144/Javeriana.upsy17-1.caer

\title{
Cuestionario de Ansiedad Estado Rasgo (STAI): análisis psicométrico y funcionamiento en una muestra de drogodependientes y controles*
}

State Trait Anxiety Inventory (STAI): Psychometric Analysis and Functioning in a Sample of Drug Addicts and Controls

Francisco JaVier del Río Olvera a

Instituto Andaluz de Sexología y Psicología, España

ORCID: http://orcid.org/0000-0002-9488-7639

Francisco Cabello Santamaría

Instituto Andaluz de Sexología y Psicología, España

Marina A. Cabello García

Instituto Andaluz de Sexología y Psicología, España

Jerónimo Aragón Vela

Universidad de Granada, España

a Autor de correspondencia. Correo electrónico: fjdelrio@iasexologia.com

Para citar este artículo: Del Río Olvera, F. J., Cabello Santamaría, F., Cabello García, M. A., \& Aragón Vela, J. (2018). Cuestionario de Ansiedad Estado Rasgo (STAI): análisis psicométrico y funcionamiento en una muestra de drogodependientes y controles. Universitas Psychologica, 17(1), 1-10. https://doi.org/1 0.11144/Javeriana.upsy17-1.caer

\begin{abstract}
RESUMEN
La presente investigación pretende evaluar las propiedades psicométricas del cuestionario STAI en población drogodependiente y comparar sus resultados con población no clínica. Se realizó un muestreo por conglomerados en 28 centros de tratamiento para las adicciones, repartidos en 27 provincias españolas. La muestra final estuvo compuesta por 1054 personas consumidoras de sustancias y 211 no consumidoras. El análisis de la fiabilidad mostró adecuada validez interna. El análisis de los ítems indica la necesidad de revisar dos de los ítems cuando se utiliza en población con historial de consumo de sustancias adictivas. Los resultados muestran que las personas con un historial de drogodependencia obtuvieron una puntuación media superior a las personas no drogodependientes, que las mujeres consiguieron una puntuación media superior a los hombres y que las personas que habían consumido sustancias depresoras lograron una puntuación superior a las que habían consumido sustancias estimulantes. Las diferencias fueron estadísticamente significativas.

Palabras clave

STAI; drogas; análisis psicométrico; conglomerados.
\end{abstract}

\section{ABSTRACT}

This investigation intends to assess the psychometric properties of the questionnaire STAI in a sample of drug abusers versus a non-clinic sample. It was carried out a sampling for conglomerates in 28 treatment centers for addiction, distributed in 27 Spanish provinces. The sample was composed by 1054 subjects who had a history of substance use and 211 with no history of substance abuse. The analysis of reliability showed an internal appropriate validity. The items analysis shows the necessity to revise two 
Francisco Javier del Río Olvera, Francisco Cabello Santamaría, Marina A. Cabello García, et al.

items when the questionnaire is used with drug abusers population. The results show that people with a history of consumption obtained a higher mean score than those who had not used substances, women obtained a higher mean score than males, and that people who had used depressants obtained a score higher than those who had consumed stimulants. The differences were statistically significant.

Keywords

STAI; drugs; psychometric analysis; conglomerates.

La ansiedad es un concepto difícil de definir sin confundirlo con otros términos como estrés o angustia (Sierra, Ortega, \& Zubeidat, 2003). La característica principal de la ansiedad es su carácter anticipatorio, en la que puede señalar un peligro, teniendo una clara función activadora de la respuesta del individuo como mecanismo adaptativo de protección (Miguel-Tobal, 1996). Su exceso puede suponer manifestaciones patológicas en el individuo, tanto a nivel emocional como a nivel funcional (Vila, 1984) como en el área sexual (Del Río, Cabello, \& Cabello, 2015; Del Río, CabelloSantamaría, Cabello-García, \& Aragón-Vela, 2017). Actualmente, es uno de los problemas psicológicos con mayor prevalencia (GuillénRiquelme \& Buela-Casal, 2011), siendo la causa del $26.1 \%$ de las consultas formales de psiquiatría (Alonso et al., 2004).

En relación con la ansiedad y con el consumo de sustancias, cabe señalar que la ansiedad es uno de los síntomas más claros durante el síndrome de abstinencia al abandonar el consumo (Pérez, 2011). Además, investigaciones previas indican que mayores niveles de ansiedad están correlacionados con el consumo de sustancias en general (Dorard et al., 2017; Scorzelli \& Chaudhry, 2009). En un estudio con 68 pacientes, Hearon et al. (2011) encontraron que las mujeres con niveles elevados de ansiedad son más propensas a consumir sedantes que los hombres con los mismos niveles. Por otra parte, si se analiza la ansiedad en relación con el tratamiento de deshabituación, algunos autores observan cómo los niveles de ansiedad son mayores antes de comenzar el tratamiento para la deshabituación de sustancias que cuando este ha finalizado (Marqueta, Jiménez-Muro, Beamonte, Gargallo, \& Nerín, 2010); el mismo efecto se produce en la familia (Bisetto, González, \& Botella, 2016).

El objetivo del presente estudio es analizar las propiedades psicométricas del Cuestionario de Ansiedad Estado Rasgo (STAI) en población con historial de adicciones. Se espera que los índices psicométricos del instrumento sean adecuados. Además, se compararán los resultados obtenidos en el cuestionario con población sin historial de consumo de sustancias, entre sexos y también se comparará la puntuación media en función del tipo de sustancia adictiva consumida. Atendiendo a investigaciones previas, se espera que las personas que han consumido sustancias adictivas obtengan una puntuación media superior en ansiedad que las personas que no han consumido dichas sustancias (Marqueta et al., 2010; Pérez, 2011; Scorzelli \& Chaudhry, 2009), que las mujeres obtengan una puntuación media superior a la de los hombres (GuillénRiquelme \& Buela-Casal, 2011; Hearon et al., 2011) y que las personas que han consumido sustancias depresoras presenten más ansiedad que aquellas que han consumido sustancias estimulantes (Cano-Vindel, MiguelTobal, González, \& Iruarrizaga, 1994).

\section{Método}

\section{Participantes}

Se utilizaron dos muestras, una correspondiente a personas con historial de abuso de drogas (1054 participantes) y otra de personas no consumidoras (211). Ambas muestras fueron seleccionadas de 27 provincias españolas. En el grupo de consumidores el rango de edad fue de entre 18 y 61 años $(M=34.58$ y $D E=$ 7.73), en el momento de participar en el estudio tenían pareja $505(47.91 \%)$ personas, con una M de 8.3 años de vida en común y 549 (52.09 \%) no la tenían, siendo hombres el $87.76 \%$ (925) y mujeres el $12.24 \%$ (129). En el grupo de no consumidores de droga, el rango de edad osciló entre 19 y 61 años $(M=35.12$ y $D E$ 
= 8.39), tenían pareja 176 (83.41\%) personas, con una media de 9.8 años de vida en pareja, y 35 (16.59\%) no la tenían, siendo el $38.86 \%$ hombres (82) y el $61.14 \%$ mujeres (129). Las diferentes sustancias adictivas que consumían los participantes antes de comenzar el tratamiento de desintoxicación se indican en la Tabla 1.

\section{Tabla 1}

Tipo de sustancia consumida por la muestra de consumidores

\begin{tabular}{lrr}
\hline \multicolumn{1}{c}{ Sustancia } & \multicolumn{1}{c}{ N } & \multicolumn{1}{c}{$\%$} \\
\hline Cocaína & 527 & 50 \\
Alcohol & 151 & 14.33 \\
Heroína & 50 & 4.74 \\
Cannabis & 26 & 2.47 \\
Fármacos estimulantes & 19 & 1.8 \\
Fármacos depresores & 5 & 0.47 \\
Cocaína y alcohol & 115 & 10.91 \\
Cocaína y heroína & 90 & 8.54 \\
3 o más sustancias & 71 & 6.74 \\
\hline Total & 1054 & 100 \\
\hline
\end{tabular}

\section{Instrumentos}

Para esta investigación, se utilizó el Cuestionario de Ansiedad Estado Rasgo ([STAI]; Spielberger, Gorsuch, \& Lushene), que está diseñado específicamente para medir la ansiedad. Tiene dos escalas de autoevaluación para medir dos conceptos independientes de la ansiedad: estado y rasgo. La ansiedad estado la definen los autores como una condición emocional transitoria del organismo, caracterizada por sentimientos subjetivos de tensión y aprensión. La ansiedad rasgo está definida como una propensión ansiosa estable que hace percibir a las personas y las situaciones como amenazadoras, elevando así la ansiedad. Tanto la escala estado como la escala rasgo tienen 20 ítems cada una, que se puntúan en una escala tipo Likert con cuatro opciones de respuesta (de 0 a 3). El cuestionario tiene una buena consistencia interna en la adaptación española, entre 0.9 y 0.93 en la ansiedad/estado y entre 0.84 y 0.87 en ansiedad/rasgo (Spielberger et al., 1982).

\section{Procedimiento}

La muestra fue recogida en 28 centros de tratamiento, mediante un muestreo por conglomerados no aleatorio. El cuestionario fue administrado en los diferentes centros por personal cualificado. En todos los casos se informó a los participantes que la colaboración era voluntaria y anónima. Para su inclusión en el estudio, los participantes debían ser mayores de edad y aceptar voluntariamente su participación. Aun así, se excluyeron las personas que presentaron cualquier patología psíquica catalogada en el DSM-IV-TR (American Psychiatric Association [APA], 2002), a excepción de la adicción a sustancias, déficit del control de impulsos y disfunciones sexuales y también a las personas que estaban tomando alguna medicación.

\section{Análisis de datos}

Para la comprobación de las hipótesis, se realizaron distintos análisis. Se estudió la fiabilidad de la escala mediante el coeficiente alfa de Cronbach. El estudio de la estructura factorial del cuestionario se llevó a cabo mediante el método de extracción de componentes principales y la rotación Varimax, verificando previamente los supuestos para dicho análisis mediante la prueba de Kaiser-Meyer-Olkin $(\mathrm{KMO})$ y la prueba de la esfericidad de Bartlett. Dado que las variables utilizadas son ordinales, la factorización se realizó a partir de una matriz con correlaciones policóricas. Para el análisis de los ítems, se calculó la correlación ítem-total, además de la media y la desviación estándar en cada uno de ellos. Antes de la comparación de los datos de ambos grupos se llevó a cabo la prueba de Kolmogorov-Smirnov. Se realizaron las pruebas $U$ de Mann-Whitney, la prueba de Kruskal-Wallis y la prueba de Dunn, para contrastar las diferencias encontradas en las puntuaciones entre los grupos de interés. Los 
análisis de datos de este estudio se efectuaron con el programa estadístico IBM® SPSS® Statistics Versión 19.0; la prueba de Dunn, con el programa informático Microsoft ${ }^{\circledR}$ Excel ${ }^{\circledR} 2010$ y el análisis mediante correlaciones policóricas, con el programa estadístico FACTOR versión 8.1 (Lorenzo-Seva \& Ferrando, 2006).

\section{Resultados}

\section{Fiabilidad de la escala}

Se hizo un análisis de la fiabilidad del cuestionario mediante el alfa de Cronbach, para ambos grupos, tanto para la escala de ansiedad rasgo como para la escala de ansiedad estado, los resultados fueron los siguientes: grupo de consumidores, ansiedad estado $=0.902$, ansiedad rasgo $=0.885$; grupo de no consumidores, ansiedad estado $=0.914$; ansiedad rasgo $=0.89$. Los resultados superan las indicaciones de Nunnally (Nunnally \& Bernstein, 1994) sobre la puntuación mínima de referencia necesaria para los cuestionarios utilizados en investigación (valores de alfa de Cronbach superiores a 0.7), por tanto el instrumento presenta valores de fiabilidad suficiente para su utilización en la presente investigación.

\section{Análisis factorial}

A continuación, se llevaron a cabo las pruebas de Kaiser-Meyer-Olkin (KMO) y la de la esfericidad de Bartlett tanto en el grupo de consumidores $\left(\mathrm{KMO}=0.951\right.$; Bartlett: $\chi^{2} 780$ $=16330.514, \rho<0$ ) como en el grupo de no consumidores $\left(\mathrm{KMO}=0.896\right.$; Bartlett: $\chi^{2}$ $780=3962.296, \rho<0)$. En ambos casos, los resultados indican que es adecuado realizar un análisis factorial. Teóricamente, si los datos de interés tienen naturaleza ordinal, la matriz de correlaciones por estimar debería ser la matriz de correlaciones policóricas, en tanto en cuanto esta estima la relación lineal entre dos variables latentes continuas que subyacen a dos variables observadas ordinales que son indicadores manifiestos de aquellas (Flora \& Curran, 2004). Como el cuestionario se contesta en una escala tipo Likert de cuatro opciones $(0-3)$, en la que se asume un nivel de medida ordinal, la factorización de los datos se hizo a partir de la matriz de correlaciones policóricas, tal y como recomiendan Muthén y Kaplan (1992). Para la elección del número de componentes se replicó el procedimiento utilizado por los autores en la adaptación española (Spielberger et al., 1982). Se extrajeron cuatro componentes, tanto del grupo de consumidores como del grupo de no consumidores, utilizando el método de componentes principales y la rotación Varimax. Los autovalores para los componentes del grupo de consumidores fueron 13.222, 3.330, $2.712 \mathrm{y}$ 1.324, explicando el $51.47 \%$ de la varianza total y los autovalores para los componentes del grupo de no consumidores fueron 15.250, 2.640, 2.118 y 1.783 , explicando el $54.48 \%$ de la varianza total. Los porcentajes de varianza explicada son similares a los obtenidos por Guillén-Riquelme y Buela-Casal (2011), en un reciente estudio. Las saturaciones de los ítems de ambos grupos se pueden observar en la Tabla 2. Se han establecido como punto de corte para la asignación de los ítems a los componentes saturaciones iguales o superiores a 0.3.

En los componentes del grupo de consumidores se observa que casi todos los ítems negativos (excepto el ítem 1) se encuentran en el componente 2, además de un ítem positivo (el ítem 32). Esto podría considerarse como un artefacto metodológico. El componente 1 tiene los ítems positivos de la escala ansiedad rasgo, y en los componentes 3 y 4 se reparten el resto de los ítems positivos de la escala ansiedad estado. En el componente 4 además se incluye el ítem 1, que también es un ítem negativo. Finalmente hay que señalar que los ítems 13 y 22 no saturan en ninguno de los componentes. Los componentes del grupo de no consumidores son similares a los anteriormente descritos, ya que en el componente 2 también se encuentran la mayoría de los ítems negativos del cuestionario (a excepción de los ítems 11, 27 y 39), en el componente 1 solo saturan ítems de la escala ansiedad rasgo, y en el componente 4 la mayoría 
son de la escala ansiedad estado (excepto el ítem 21). Donde más se aprecia la diferencia entre ambos grupos es en el componente 3 , ya que en el grupo de no consumidores saturan ítems de ambas escalas, tanto positivos como negativos.

\section{Tabla 2}

Matriz de saturaciones de los ítems en los componentes rotados en el grupo de consumidores y de no consumidores

\begin{tabular}{|c|c|c|c|c|c|c|c|c|}
\hline \multirow{2}{*}{ Ítem } & \multicolumn{4}{|c|}{ Grupo de consumidores } & \multicolumn{4}{|c|}{ Grupo de no consumidores } \\
\hline & $\mathrm{C} 1$ & $\mathrm{C} 2$ & $\mathrm{C} 3$ & $\mathrm{C} 4$ & $\mathrm{C} 1$ & $\mathrm{C} 2$ & C3 & $\mathrm{C} 4$ \\
\hline 1 & 0.05 & 0.04 & -0.13 & -0.44 & 0.04 & 0.73 & 0.01 & -0.18 \\
\hline 2 & 0.04 & 0.59 & -0.18 & -0.05 & -0.02 & 0.62 & -0.23 & 0.06 \\
\hline 3 & 0.12 & -0.06 & 0.45 & 0.41 & 0.13 & -0.45 & 0.05 & 0.42 \\
\hline 4 & 0.06 & -0.15 & 0.50 & 0.10 & 0.12 & -0.44 & 0.11 & 0.37 \\
\hline 5 & 0.12 & 0.54 & -0.16 & -0.18 & -0.07 & 0.74 & -0.01 & -0.06 \\
\hline 6 & 0.17 & -0.07 & 0.48 & 0.36 & 0.15 & -0.35 & 0.01 & 0.58 \\
\hline 7 & 0.17 & 0.07 & 0.63 & -0.14 & 0.07 & -0.04 & 0.47 & 0.32 \\
\hline 8 & 0.07 & 0.35 & -0.12 & -0.16 & -0.20 & 0.50 & 0.19 & -0.09 \\
\hline 9 & 0.07 & -0.01 & 0.70 & -0.06 & 0.11 & 0.03 & 0.52 & 0.49 \\
\hline 10 & 0.15 & 0.60 & -0.19 & -0.11 & -0.03 & 0.74 & -0.01 & 0.04 \\
\hline 11 & 0.01 & 0.51 & -0.29 & 0.27 & 0.08 & 0.09 & -0.77 & 0.00 \\
\hline 12 & 0.15 & -0.07 & 0.48 & 0.32 & 0.23 & -0.09 & 0.22 & 0.61 \\
\hline 13 & 0.23 & 0.02 & 0.17 & 0.20 & 0.14 & -0.19 & 0.27 & 0.38 \\
\hline 14 & 0.07 & -0.03 & 0.70 & -0.16 & -0.02 & 0.06 & 0.65 & 0.41 \\
\hline 15 & 0.09 & 0.51 & -0.23 & -0.27 & -0.21 & 0.72 & 0.20 & -0.20 \\
\hline 16 & 0.15 & 0.64 & -0.28 & 0.14 & 0.24 & 0.31 & -0.62 & -0.09 \\
\hline 17 & 0.11 & -0.03 & 0.61 & -0.05 & 0.19 & -0.25 & 0.19 & 0.33 \\
\hline 18 & 0.17 & 0.02 & 0.61 & 0.08 & 0.25 & -0.27 & 0.17 & 0.47 \\
\hline 19 & 0.09 & 0.79 & 0.03 & -0.04 & 0.15 & 0.78 & -0.20 & 0.27 \\
\hline 20 & 0.14 & 0.78 & -0.08 & -0.11 & 0.09 & 0.80 & -0.18 & 0.10 \\
\hline 21 & -0.26 & 0.72 & 0.18 & -0.05 & -0.08 & 0.43 & -0.40 & 0.35 \\
\hline 22 & 0.29 & -0.12 & 0.12 & -0.05 & 0.64 & 0.03 & 0.02 & -0.06 \\
\hline 23 & 0.50 & 0.02 & 0.18 & -0.02 & 0.56 & 0.10 & 0.27 & 0.08 \\
\hline 24 & 0.51 & -0.05 & 0.18 & -0.09 & 0.02 & -0.40 & 0.25 & -0.06 \\
\hline 25 & 0.49 & 0.00 & 0.14 & -0.09 & 0.23 & -0.01 & 0.34 & -0.06 \\
\hline 26 & -0.1 & 0.56 & 0.03 & -0.12 & -0.51 & 0.41 & 0.08 & 0.26 \\
\hline 27 & -0.18 & 0.38 & 0.06 & -0.11 & -0.71 & 0.10 & -0.01 & 0.17 \\
\hline 28 & 0.62 & -0.17 & 0.05 & -0.06 & 0.37 & -0.00 & 0.40 & 0.00 \\
\hline 29 & 0.72 & 0.09 & -0.06 & 0.08 & 0.43 & 0.24 & 0.50 & -0.01 \\
\hline 30 & -0.26 & 0.70 & 0.14 & 0.03 & 0.03 & 0.49 & -0.45 & 0.27 \\
\hline 31 & 0.44 & 0.28 & -0.07 & 0.28 & 0.64 & 0.00 & -0.13 & -0.09 \\
\hline 32 & 0.52 & -0.31 & -0.00 & -0.14 & 0.13 & 0.00 & 0.65 & -0.25 \\
\hline 33 & -0.22 & 0.65 & 0.04 & 0.21 & -0.17 & 0.23 & -0.62 & 0.27 \\
\hline 34 & 0.37 & -0.19 & 0.02 & -0.16 & 0.14 & 0.09 & 0.58 & -0.06 \\
\hline 35 & 0.56 & -0.18 & 0.14 & -0.01 & 0.42 & 0.03 & 0.31 & 0.01 \\
\hline 36 & -0.18 & 0.76 & 0.09 & 0.10 & -0.13 & 0.33 & -0.54 & 0.28 \\
\hline 37 & 0.66 & -0.01 & -0.04 & 0.09 & 0.46 & 0.04 & 0.33 & 0.05 \\
\hline 38 & 0.59 & 0.03 & 0.00 & 0.05 & 0.52 & -0.10 & 0.19 & -0.08 \\
\hline 39 & -0.23 & 0.60 & 0.10 & 0.10 & -0.42 & 0.07 & -0.38 & 0.22 \\
\hline 40 & 0.56 & -0.07 & 0.11 & 0.00 & 0.63 & -0.10 & -0.01 & -0.04 \\
\hline
\end{tabular}

Análisis de los ítems

Para el análisis de los ítems, se procedió al cálculo de la correlación ítem-total corregida en los dos grupos, habiéndose modificado previamente el sentido de la puntuación de los ítems inversos (Tabla 3). Siguiendo las indicaciones de Ebel (1965), los ítems con una correlación ítemtotal por debajo de 0.19 deben eliminarse, y los que se encuentren entre 0.2 y 0.29 deben revisarse. Según estas indicaciones en el grupo de consumidores se debería eliminar el ítem 31 ("Suelo tomar las cosas demasiado en serio") y se debería revisar el ítem 13 ("Estoy desasosegado"). En cambio, en el grupo de no consumidores no sería necesario revisar ni eliminar ninguno de los ítems. El hecho de que fuera necesario modificar dos ítems en un grupo y no en el otro hace pensar en la diferencia en la forma de responder al cuestionario por parte de ambos grupos, lo que podría ser una consecuencia de la historia de consumo.

Tabla 3

Análisis de los ítems en los dos grupos

\begin{tabular}{|c|c|c|c|c|c|c|c|c|}
\hline \multirow{2}{*}{ Ítem } & \multicolumn{4}{|c|}{ Consumidores $(N=1054)$} & \multicolumn{4}{|c|}{ No consumidores $(N=211)$} \\
\hline & $M^{M}$ & $D E^{2}$ & $r^{3}$ & $\alpha^{4}$ & $M^{2}$ & $D E^{2}$ & $r^{3}$ & $\alpha^{4}$ \\
\hline 1 & 1.13 & 0.81 & 0.56 & 0.89 & 0.86 & 0.71 & 0.62 & 0.90 \\
\hline 2 & 1.09 & 0.83 & 0.58 & 0.89 & 0.79 & 0.67 & 0.60 & 0.90 \\
\hline 3 & 0.75 & 0.83 & 0.61 & 0.89 & 0.45 & 0.68 & 0.63 & 0.90 \\
\hline 4 & 0.76 & 0.90 & 0.55 & 0.89 & 0.35 & 0.64 & 0.59 & 0.90 \\
\hline 5 & 1.09 & 0.94 & 0.55 & 0.89 & 0.71 & 0.69 & 0.65 & 0.90 \\
\hline 6 & 0.63 & 0.84 & 0.61 & 0.89 & 0.3 & 0.64 & 0.55 & 0.91 \\
\hline 7 & 1.11 & 1.03 & 0.44 & 0.90 & 0.54 & 0.78 & 0.49 & 0.91 \\
\hline 8 & 1.49 & 0.96 & 0.38 & 0.90 & 1.47 & 0.89 & 0.44 & 0.91 \\
\hline 9 & 0.77 & 0.89 & 0.55 & 0.89 & 0.46 & 0.78 & 0.55 & 0.91 \\
\hline 10 & 1.30 & 0.85 & 0.58 & 0.89 & 1 & 0.69 & 0.61 & 0.90 \\
\hline 11 & 1.08 & 0.93 & 0.47 & 0.89 & 0.84 & 0.76 & 0.49 & 0.91 \\
\hline 12 & 0.78 & 0.85 & 0.61 & 0.89 & 0.44 & 0.69 & 0.59 & 0.90 \\
\hline 13 & 0.87 & 0.90 & 0.25 & 0.90 & 0.44 & 0.74 & 0.47 & 0.91 \\
\hline 14 & 0.92 & 0.96 & 0.51 & 0.89 & 0.34 & 0.65 & 0.48 & 0.91 \\
\hline 15 & 1.29 & 0.94 & 0.63 & 0.89 & 1.1 & 0.81 & 0.66 & 0.90 \\
\hline 16 & 1.16 & 0.92 & 0.56 & 0.89 & 0.9 & 0.68 & 0.56 & 0.90 \\
\hline 17 & 1.13 & 0.94 & 0.52 & 0.89 & 0.72 & 0.77 & 0.57 & 0.90 \\
\hline 18 & 0.61 & 0.84 & 0.53 & 0.89 & 0.17 & 0.44 & 0.54 & 0.91 \\
\hline 19 & 1.23 & 0.89 & 0.53 & 0.89 & 0.98 & 0.68 & 0.50 & 0.91 \\
\hline 20 & 0.91 & 0.84 & 0.62 & 0.89 & 0.72 & 0.64 & 0.65 & 0.90 \\
\hline 21 & 1.14 & 0.79 & 0.59 & 0.87 & 0.62 & 0.62 & 0.54 & 0.88 \\
\hline 22 & 0.96 & 0.84 & 0.38 & 0.88 & 0.69 & 0.70 & 0.40 & 0.88 \\
\hline 23 & 0.91 & 0.80 & 0.45 & 0.88 & 0.56 & 0.60 & 0.51 & 0.88 \\
\hline 24 & 1.51 & 1.04 & 0.52 & 0.87 & 0.56 & 0.66 & 0.39 & 0.88 \\
\hline 25 & 1.21 & 0.90 & 0.44 & 0.88 & 0.75 & 0.70 & 0.39 & 0.88 \\
\hline 26 & 1.51 & 0.92 & 0.44 & 0.88 & 1.2 & 0.80 & 0.54 & 0.88 \\
\hline 27 & 1.58 & 1.01 & 0.37 & 0.88 & 1.07 & 0.95 & 0.56 & 0.88 \\
\hline 28 & 1.19 & 0.91 & 0.63 & 0.87 & 0.55 & 0.61 & 0.53 & 0.88 \\
\hline 29 & 1.41 & 0.97 & 0.49 & 0.88 & 0.85 & 0.77 & 0.49 & 0.88 \\
\hline 30 & 1.27 & 0.95 & 0.60 & 0.87 & 0.55 & 0.69 & 0.53 & 0.88 \\
\hline 31 & 1.67 & 0.88 & 0.14 & 0.89 & 1.45 & 0.79 & 0.35 & 0.89 \\
\hline 32 & 1.32 & 0.96 & 0.61 & 0.87 & 0.69 & 0.72 & 0.48 & 0.88 \\
\hline 33 & 1.37 & 0.94 & 0.55 & 0.87 & 0.9 & 0.81 & 0.71 & 0.87 \\
\hline 34 & 1.24 & 0.95 & 0.40 & 0.88 & 0.53 & 0.67 & 0.38 & 0.88 \\
\hline 35 & 1.08 & 0.84 & 0.62 & 0.87 & 0.58 & 0.68 & 0.45 & 0.88 \\
\hline 36 & 1.3 & 0.92 & 0.58 & 0.87 & 0.73 & 0.65 & 0.65 & 0.88 \\
\hline 37 & 1.22 & 0.88 & 0.51 & 0.87 & 0.6 & 0.69 & 0.52 & 0.88 \\
\hline 38 & 1.43 & 1.02 & 0.45 & 0.88 & 0.73 & 0.82 & 0.57 & 0.88 \\
\hline 39 & 1.41 & 0.94 & 0.52 & 0.87 & 0.64 & 0.73 & 0.55 & 0.88 \\
\hline 40 & 1.56 & 0.96 & 0.55 & 0.87 & 1 & 0.77 & 0.52 & 0.88 \\
\hline
\end{tabular}

Análisis de las puntuaciones en los grupos

La prueba de Kolmogorov-Smirnov señaló que la muestra no cumple el supuesto de normalidad (ansiedad estado: $Z=3.016$, sig. $=0$; ansiedad rasgo: $Z=1.77$, sig. $=0.004$ ), por tanto se utilizaron pruebas no paramétricas para el análisis de las diferencias entre los grupos. Se calculó la puntuación media de las dos escalas del cuestionario en ambos grupos (grupo consumidores: ansiedad estado $=20.349$, ansiedad rasgo $=26.341$; grupo 
no consumidores: ansiedad estado $=13.521$, ansiedad rasgo $=15.246$ ), sin diferenciar por sexos, y se verificó si la diferencia existente entre ellos era o no significativa, utilizando la prueba $U$ de Mann-Whitney. Los resultados señalan que la diferencia es estadísticamente significativa, tanto en ansiedad estado $(\rho=0)$ como en ansiedad rasgo $(\rho=0)$.

Se analizó también la influencia del sexo en la puntuación media en el cuestionario; para ello, se calculó la puntuación media obtenida por los hombres y por las mujeres, comparándose entre ellos en función de cada grupo. Se analizó en primer lugar la diferencia encontrada entre sexos dentro del grupo de consumidores (Tabla 4), observándose que las mujeres obtienen una puntuación media superior a los hombres tanto en la escala ansiedad estado como en la escala ansiedad rasgo. Tras realizar la prueba U de Mann-Whitney, se comprobó que dicha diferencia es estadísticamente significativa en ambas escala. Resultados similares se obtuvieron en el grupo de no consumidores (Tabla 4).

\section{Tabla 4}

Análisis de las diferencias de puntuaciones medias entre hombres y mujeres en cada uno de los grupos, en ambas escalas del cuestionario, mediante la $U$ de Mann-Whitney

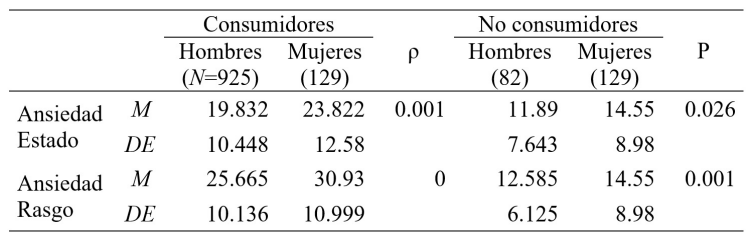

Posteriormente, se analizó de nuevo la diferencia entre ambos grupos del estudio, pero en esta ocasión teniendo en cuenta la variable sexo, es decir, comparando los hombres consumidores con los no consumidores, y realizando la misma comparativa entre las mujeres (Tabla 5). En ambos casos, las personas consumidoras obtenían una puntuación media superior, siendo dicha diferencia estadísticamente significativa al emplear la prueba de U de Mann-Whitney.

\section{Tabla 5}

Análisis de las diferencias de puntuaciones medias entre los hombres y las mujeres de cada grupo, en las escalas del cuestionario STAI mediante la prueba U de Mann-Whitney

\begin{tabular}{|c|c|c|c|c|c|c|c|}
\hline \multirow[b]{2}{*}{ Escalas } & & \multicolumn{2}{|c|}{ Hombres } & \multirow[b]{2}{*}{$\rho$} & \multicolumn{2}{|c|}{ Mujeres } & \\
\hline & & $\begin{array}{c}\text { Grupo } \\
\text { Consumidor } \\
(N=925)\end{array}$ & $\begin{array}{l}\text { Grupo no } \\
\text { consumidor } \\
(82)\end{array}$ & & $\begin{array}{c}\text { Grupo } \\
\text { Consumidor } \\
(129)\end{array}$ & $\begin{array}{l}\text { Grupo no } \\
\text { consumidor } \\
(129)\end{array}$ & $\mathrm{P}$ \\
\hline Ansiedad & $M$ & 19.83 & 11.89 & 0 & 23.82 & 14.55 & 0 \\
\hline Estado & $D E$ & 10.44 & 7.64 & & 12.58 & 8.98 & \\
\hline Ansiedad & $M$ & 25.66 & 12.58 & 0 & 30.93 & 14.55 & 0 \\
\hline Rasgo & $D E$ & 10.13 & 6.12 & & 10.99 & 8.98 & \\
\hline
\end{tabular}

A continuación, se analiza la relación existente entre las escalas del STAI y el tipo de sustancia consumida. Según los efectos de las sustancias en el sistema nervioso central, se pueden clasificar en estimulantes, depresoras o perturbadoras (Escohotado, 2001). Se ha realizado la clasificación de la siguiente forma: los que habían consumido cocaína o psicofármacos estimulantes se incluyeron dentro del grupo de sustancias estimulantes, los que habían consumido heroína, alcohol o psicofármacos depresores en el grupo de sustancias depresoras y los que habían consumido cannabis en el grupo de sustancias perturbadoras, dejando fuera de este análisis a las personas no consumidoras y a las que habían consumido alguna mezcla de sustancias que no pudiera ser incluida en ninguno de estos tres grupos (cocaína y alcohol, cocaína y heroína, 3 o más sustancias). En la Tabla 6, se presentan las puntuaciones medias y las desviaciones estándar obtenidas en el cuestionario. Se observa que las personas que obtienen una puntuación media mayor tanto en ansiedad estado como en ansiedad rasgo son aquellas que habían consumido sustancias depresoras, seguidas por las que habían consumido sustancias perturbadoras y finalmente quienes habían consumido sustancias estimulantes. Esto puede ser debido al conocido como efecto rebote (Lorenzo, Ladero, Leza, \& Lizasoain, 2009; Mendoza, 2008), de forma que las sustancias que provocan más ansiedad al ser consumidas, tras su abandono, provocan una disminución notable de la ansiedad en la persona que las consumía, y ocurriría lo contrario con las sustancias depresoras, que tras su abandono, 
harían que en la persona consumidora aumentara los niveles de ansiedad.

\section{Tabla 6}

Puntuaciones medias y desviaciones estándar en las escalas del cuestionario STAI (Ansiedad Estado y Ansiedad Rasgo), según los efectos de la droga en el sistema nervioso central

\begin{tabular}{|c|c|c|c|c|}
\hline & & & $\begin{array}{c}\text { Ansiedad } \\
\text { estado }\end{array}$ & $\begin{array}{c}\text { Ansiedad } \\
\text { rasgo }\end{array}$ \\
\hline \multirow{6}{*}{$\begin{array}{l}\text { Efecto en } \\
\text { el SNC }\end{array}$} & \multirow[t]{2}{*}{ Sustancias estimulantes } & $M$ & 19.35 & 25.19 \\
\hline & & $D E$ & 10.46 & 10.13 \\
\hline & \multirow[t]{2}{*}{ Sustancias depresoras } & $M$ & 21.64 & 28.46 \\
\hline & & $D E$ & 10.99 & 10.22 \\
\hline & \multirow[t]{2}{*}{ Sustancias perturbadoras } & $M$ & 19.92 & 27.04 \\
\hline & & $D E$ & 12.36 & 10.75 \\
\hline
\end{tabular}

Para contrastar si las diferencias encontradas en las puntuaciones medias en las dos escalas de ansiedad eran estadísticamente significativas, se realizó la prueba de Kruskal-Wallis. El resultado señala que las diferencias son estadísticamente significativas $\left(\chi^{2}=6.959\right.$; gl $=2$; Sig. Asintót. $=0.031)$. La prueba de Kruskal-Wallis no señala entre qué grupos se encuentra dicha diferencia, para ello es necesario efectuar una prueba adicional, la prueba de Dunn (Rial \& Varela, 2008), la cual señaló que la diferencia significativa se encontraba entre las sustancias estimulantes $(M=19.35)$ y las sustancias depresoras $(M=21.64)$.

Para verificar si la diferencia obtenida en la media en la escala ansiedad rasgo en función del tipo de sustancia era estadísticamente significativa, se siguió el mismo procedimiento que con la escala ansiedad estado. La prueba de Kruskal-Wallis señala que sí existen diferencias estadísticamente significativas entre los grupos $\left(\chi^{2}=15.659 ; g l=2\right.$; Sig. Asintót. $\left.=0\right)$. Según la prueba de Dunn, la diferencia se encuentra entre las personas que han consumido sustancias estimulantes $(M=25.19)$ y las que han consumido sustancias depresoras $(\mathrm{M}=$ 28.46), igual que se observaba en el caso de la escala ansiedad estado.

\section{Discusión}

Los resultados del alfa de Cronbach muestran que la fiabilidad del cuestionario en cada una de las escalas es adecuada en ambos grupos, lo que hace suponer una adecuada consistencia interna. Estos datos son similares a los obtenidos por los autores del cuestionario (Spielberger et al., 1982), así como por el reciente análisis del cuestionario llevado a cabo por GuillénRiquelme y Buela-Casal (2011; ansiedad rasgo $=0.9$, ansiedad estado $=0.94)$. En este primer análisis, ya se puede observar un dato que se repetirá a lo largo del resto, y es que los resultados del grupo de consumidores y del grupo de no consumidores serán ligeramente diferentes, obteniendo estos últimos mejores datos psicométricos.

La varianza explicada en cada uno de los grupos es ligeramente superior al $50 \%$ (consumidores $=51.47 \%$; no consumidores $=54.48 \%)$. En la extracción de los componentes se replicó el análisis de datos original (Spielberger et al., 1982), y aunque los datos no replican exactamente los encontrados por los autores del cuestionario, muestran con claridad la estructura tetrafactorial hallada en investigaciones anteriores (Guillén-Riquelme \& Buela-Casal, 2011; Vera-Villarroel, Buela-Casal, \& Spielberger, 2007).

El análisis de los ítems indica que en el grupo de no consumidores todos los ítems tienen una adecuada correlación ítem-total, por lo que no sería necesario modificar o eliminar ninguno de ellos. En cambio, en el grupo de consumidores, sería necesario modificar el ítem $13(r=0.259)$ y eliminar el ítem $31(r=0.146)$. Para los autores de esta investigación es llamativa esta diferencia entre ambos grupos, y hace pensar en la principal diferencia entre ellos, el consumo de sustancias adictivas. Podría ser precipitado suponer que sería necesario redefinir ambos ítems para utilizar adecuadamente el cuestionario en población drogodependiente, pero a la vista de estos resultados sería recomendable replicar este estudio con población drogodependiente para verificar los resultados. Además, se deberían tener en cuenta otras variables que podrían haber influido en estos resultados, como puede ser por ejemplo el nivel de estudios, para así aislar en mayor medida el efecto del consumo de sustancias. 
Al analizar las puntuaciones obtenidas en el cuestionario por los diferentes grupos, se observa cómo las personas consumidoras obtienen una puntuación media superior a las obtenidas por las personas no consumidoras, tanto en la escala ansiedad estado $(20.349>13.521)$ como en la escala ansiedad rasgo $(26.341>15.246)$, siendo mayor la diferencia en esta última. La prueba estadística U de Mann-Whitney señala que la diferencia encontrada entre ambos grupos es estadísticamente significativa. Esto es coincidente con estudios previos que señalan que personas que han consumido sustancias presentan de media niveles más elevados de ansiedad (Marqueta et al., 2010; Pérez, 2011; Scorzelli \& Chaudhry, 2009; Wright, Scerpella, \& Lisdahl, 2016).

Al mismo tiempo, también se observa que la variable sexo afecta al nivel de ansiedad, presentando las mujeres mayores niveles de ansiedad media que los hombres, tanto en ansiedad estado (mujeres: consumidoras = 23.822, no consumidoras $=14.558$; hombres: consumidores $=19.832$, no consumidores $=$ 11.890), como en ansiedad rasgo (mujeres: consumidoras $=30.93$, no consumidoras $=$ 16.946; hombres: consumidores $=25.665$, no consumidores $=12.585)$, tal y como indicaban estudios previos (Guillén-Riquelme \& BuelaCasal, 2011; Hearon et al., 2011; Spielberger et al., 1982).

Es destacable también señalar cómo las personas que han consumido sustancias depresoras obtienen una puntuación media superior en ambas escalas del cuestionario que las personas que han consumido sustancias estimulantes, como también se observa en la experiencia clínica diaria. Este hecho podría ser debido al efecto rebote (Lorenzo et al., 2009; Mendoza, 2008), aunque se debería profundizar en más investigación para verificar dicha hipótesis.

Hasta ahora, se sabía que las personas drogodependientes presentaban niveles de ansiedad más elevados que la población no consumidora, ya que es un síntoma del que los propios pacientes informan, pero del que no había datos objetivos. La principal aportación de este trabajo es la de poder establecer un punto de corte a partir del cual decidir qué niveles de ansiedad pueden considerarse aceptables en población drogodependiente. Esto puede permitir a los profesionales que trabajan en los centros de tratamiento de las adicciones saber cuándo deben realizar una intervención terapéutica encaminada al control de la ansiedad.

Como conclusión, cabe señalar que la escala presenta buenas propiedades psicométricas en población drogodependiente, tanto en la escala de ansiedad rasgo como en la de ansiedad estado, por tanto se considera adecuada su utilización en contextos de investigación. Por otro lado, las características psicométricas presentadas han sido mejores en la población no clínica que en la población con historial de adicciones, por tanto, se concluye que el cuestionario se ajusta mejor a población no clínica. Se recomienda en un futuro replicar la investigación para verificar las diferencias encontradas en los resultados obtenidos entre población con historial de adicciones y población sin consumo de sustancias, las diferencias encontradas entre hombres y mujeres, así como según el tipo de sustancia consumida. Al mismo tiempo, se deberían incluir más variables diferenciadoras entre ambos grupos, para dilucidar mejor las variables que influyen en dicha diferencia.

\section{Agradecimientos}

Los autores agradecen, a los siguientes centros de tratamiento, su participación en la presente investigación: Centro de Solidaridad de Zaragoza Proyecto Hombre, Centro Español de Solidaridad de Córdoba - Proyecto Hombre, Centro Español de Solidaridad Proyecto Hombre Madrid, Comunidad Terapéutico O Confurco ASFEDRO, FGSVA Proyecto Hombre Granada, Fundación Alcándara Proyecto Hombre Salamanca, Fundación Aldaba Proyecto Hombre Valladolid, Fundación Ángaro Proyecto Hombre Jaén, Fundación Arzobispo Miguel Roca Proyecto Hombre Valencia, Fundación CALS Proyecto Hombre BierzoLeón, Fundación Canaria CESICA Proyecto 
Hombre, Fundación Candeal Proyecto Hombre Burgos, Fundación Centro de Solidaridad de La Rioja Proyecto Hombre La Rioja, Fundación CESPA Proyecto Hombre Asturias, Fundación Jeiki, Fundación Proyecto Hombre Navarra, Fundación Solidaridad y Reinserción Proyecto Hombre Murcia, Projecte Home Balears, Proyecto Hombre Almería, Proyecto Hombre Cantabria, Proyecto Hombre Castilla La Mancha, Proyecto Hombre Cataluña, Proyecto Hombre Extremadura, Proyecto Hombre Huelva, Proyecto Hombre Málaga CESMA, Proyecto Hombre Provincia de Cádiz y Proyecto Hombre Sevilla.

\section{Referencias}

Alonso, J., Angermeyer, M. C., Bernert, S., Bruffaerts, R., Brugha, T. S., Bryson, H.,... Vollebergh, W. A. (2004). Use of mental health services in Europe: Results from the European Study of the Epidemiology of Mental Disorders (ESEMeD) project. Acta Psychiatrica Scandinavica, 109 (Suppl. 420), 47-54. http://dx.doi.org/10.1111/j.1600-00 47.2004.00330.x

American Psychiatric Association. (2002). DSMIV-TR. Breviario. Criterios diagnósticos. Barcelona: Masson.

Bisetto, D., González, R., \& Botella, A. (2016). Family-Based Intervention Program for parents of substance-abusing youth and adolescents. Journal of Addiction, ID 4320720. http://dx.doi.org/10.1155/2016/4 320720

Cano-Vindel, A., Miguel-Tobal, J. J., González, H., \& Iruarrizaga, I. (1994). El afrontamiento de la ansiedad en las drogodependencias. Anales de Psicología, 10(2), 145-156. Recuperado de http://www .um.es/analesps/v10/v10_2/05-10_2.pdf

Del Río, F. J., Cabello, F., \& Cabello, M. A. (2015). Relación entre disfunción eréctil y ansiedad. Revista Internacional de Andrología, 13(Especial 17 Congreso Nacional ASESA), 28. Recuperado de http://asesagrancanaria2015.com/wp-co ntent/uploads/2014/03/programa210115.p df

Del Río, F. J., Cabello-Santamaría, F., CabelloGarcía, M. A., \& Aragón-Vela, J. (2017). Sexual pain disorders in Spanish women drug users. Substance Use $\mathcal{E}$ Misuse, 52, 145-151. http://dx.doi.org/10.1080/108260 84.2016.1222625

Dorard, G., Bungener, C., Phan, O., Edel, Y., Corcos, M., \& Berthoz, S. (2017). Is alexithymia related to cannabis use disorder? Results from a case-control study in outpatient adolescent cannabis abusers. Journal of Psychosomatic Research, 95, 74-80. http://dx.doi.org/10.1016/j.jpsyc hores.2017.02.012

Ebel, R. L. (1965). Measuring educational achievement. Englewood Cliffs, NJ: Prentice Hall.

Escohotado, A. (2001). Historia general de las drogas. Madrid: Espasa Calpe.

Flora, D. B., \& Curran, P. J. (2004). An empirical evaluation of alternative methods of estimation for confirmatory factor analysis with ordinal data. Psychological Methods, 9, 466-491. http://dx.doi.org/10.1037/1082-9 89X.9.4.466

Guillén-Riquelme, A., \& Buela-Casal, G. (2011). Actualización psicométrica y funcionamiento diferencial de los ítems en el State Trait Anxiety Inventory (STAI). Psicothema, 23(3), 510-515. Recuperado de http://www.psicothema.es/pdf/3916.pdf

Hearon, B. A., Calkins, A. W., Halperin, D. M., McHugh, R. K., Murray H. W., \& Otto, M. W. (2011). Anxiety sensitivity and illicit sedative use among opiate-dependent women and men. The American Journal of Drug and Alcohol Abuse, 37, 43-47. http://d x.doi.org/10.3109/00952990.2010.535581

Lorenzo, P., Ladero, J. M., Leza, J. C., \& Lizasoain, I. (2009). Drogodependencias: farmacología, patología, psicología, legislación. Buenos Aires: Editorial Médica Panamericana.

Lorenzo-Seva, U., \& Ferrando, P. J. (2006). FACTOR: A computer program to fit the exploratory factor analysis model. Behavioral Research Methods, Instruments 
Francisco Javier del Río Olvera, Francisco Cabello Santamaría, Marina A. Cabello García, et al.

and Computers, 38(1), 88-91. http://dx.doi. org/10.3758/BF03192753

Marqueta, A., Jiménez-Muro, A., Beamonte, A., Gargallo, P., \& Nerín, I. (2010). Evolución de la ansiedad en el proceso de dejar de fumar en fumadores que acuden a una Unidad de Tabaquismo. Adicciones, 2(4), 317-324. Recuperado de http://www.adicciones.es/index.php/adi cciones/article/view/173

Mendoza, N. (2008). Farmacología médica. Buenos Aires: Editorial Médica Panamericana.

Miguel-Tobal, J. J. (1996). La ansiedad. Madrid: Santillana.

Muthén, B., \& Kaplan, D. (1992). A comparison of some methodologies for the factor analysis of non-normal Likert variables: A note on the size of the model. British Journal of Mathematical and Statistical Psychology, 38, 171-189. http://dx.doi.org/10.1111/j.20 44.8317.1985.tb00832.x

Nunnally, J. C., \& Bernstein, I. H. (1994). Psychometric theory (3. - ed.). Nueva York: McGrawHill.

Pérez, F. (2011). Estudio sobre adicciones. Perfiles de drogodependientes y eficacia del tratamiento en Proyecto Hombre Burgos. Burgos: Publicaciones de la Excma. Diputación Provincial de Burgos.

Rial, A., \& Varela, J. (2008). Estadística práctica para la investigación en ciencias de la salud. La Coruña, ES: Netbiblo, S. L.

Scorzelli, J. F., \& Chaudhry, S. Z. (2009). Relationship between anxiety and addiction to a depressant drug. Journal of Psychoactive Drugs, 41(1), 61-66. http://dx. doi.org/10.1080/02791072.2009.10400675

Sierra, J. C., Ortega, V., \& Zubeidat, I. (2003). Ansiedad, angustia y estrés: tres conceptos a diferenciar. Revista MalEstar e Subjetividade/Fortaleza, 3(1), 10-59.

Recuperado de http://pepsic.bvsalud.org/pd f/malestar/v3n1/02.pdf

Spielberger, C. D., Gorsuch, R. L., \& Lushene, R. E. (1982). Manual STAI, Cuestionario de Ansiedad Estado Rasgo. Madrid: TEA Ediciones.
Vera-Villarroel, P., Buela-Casal, G., \& Spielberger, C. D. (2007). Preliminary analysis and normative data of the State-Trait Anxiety Inventory (STAI) in adolescent and adults of Santiago, Chile. Terapia Psicológica, 25, 155-162. http://dx.doi.org/10.4067/S071848082007000200006

Vila, J. (1984). Técnicas de reducción de ansiedad. En J. Mayor \& F. J. Labrador (Eds.), Manual de modificación de conducta (pp. 229-264). Madrid: Alhambra.

Wright, N. E., Scerpella, D., \& Lisdahl, K. M. (noviembre, 2016). Marijuana use is associated with behavioral approach and depressive symptoms in adolescents and emerging adults. PLOS ONE 11, e0166005. http://dx.doi.org/10.1371/ journal.pone.0166005

\section{Notas}

* Artículo de investigación. 